\title{
CREACIÓN Y GESTIÓN DEL VALOR DE MARCA PERSONAL: PERSONAL BRANDING Y ADAPTACIÓN AL NUEVO ESCENARIO LABORAL CONTEMPORÁNEO.
}

\author{
SERGIO LÓPEZ SALAS ${ }^{1}$ \\ Personal Asociado en el Departamento de Dirección de Empresas y Sociología, área de \\ Comercialización e Investigación de Mercados. Facultad de Empresa, Finanzas y \\ Turismo. Av. de la Universidad, S/N, 10071 Cáceres (España). Universidad de \\ Extremadura. \\ MARIANO URRACO SOLANILLA ${ }^{2}$ \\ Profesor contratado doctor en el Área de Sociología. Facultad de Ciencias de la Salud y \\ de la Educación. Carretera de La Coruña, km. 38,500, 28400 Collado Villalba, Madrid \\ (España). Universidad a Distancia de Madrid (UDIMA)
}

\section{Resumen.}

Los últimos procesos de transformación económica y social han provocado un profundo cambio en el mercado laboral, dibujando un escenario de incertidumbre y dificultad creciente para acceder al empleo. Asimismo, el desarrollo tecnológico y, específicamente, el auge de las redes sociales introduce una serie de efectos sobre la forma de buscar empleo y de presentarse los individuos frente a los procesos selectivos en este ámbito. En ese contexto, los individuos necesitan resaltar por encima de otros competidores, destacando, en ese sentido, la imagen personal que se pretende construir y proyectar como factor que puede devenir crucial a la hora de lograr el éxito laboral. En esta situación, individuos particulares se convierten en gestores de su propia marca, empresarios de sí mismos en el transformado mercado de trabajo del siglo XXI.

De este modo surge una nueva disciplina de marketing, llamada "personal branding", destinada a gestionar esta marca personal. En virtud de los postulados de dicha disciplina se pueden aplicar los principios del comercio tradicional a las personas, entendidas como productos y, al mismo tiempo, como empresarios y directores de su propia acción personal (sujeto y objeto).

Este artículo trata de aplicar algunas de las principales teorías del marketing y del branding a la gestión de la marca personal. Se presenta como un ejercicio de revisión de algunos elementos y principios clásicos de la actividad empresarial, extrapolables, hoy, a los procedimientos de búsqueda de empleo llevados a cabo por los individuos. Se postula como plausible esta traslación y se apuntan aplicaciones concretas de este enfoque para el análisis del mercado laboral contemporáneo.

\section{Palabras clave:}

Redes sociales, empresarialización de sí, notoriedad, gestión de marca, empleo, selección de trabajadores.

\footnotetext{
${ }^{1}$ slopez@uemc.es

2 mariano.urraco@udima.es
} 


\title{
THE CREATION AND MANAGEMENT OF PERSONAL BRAND EQUITY: PERSONAL BRANDING AND ADAPTATION TO THE NEW AND CONTEMPORARY JOB MARKET
}

\begin{abstract}
.
The latest processes of social and economic transformation have produced a great change in the job market, creating a scenario of uncertainty and increasing difficulty for obtaining employment. Similarly, technological development and, more specifically, the rise of social networks, is causing a series of effects upon the ways in which individuals look for and apply for employment. Within this context, individuals need to stand out from other competitors, by highlighting the personal image that they intend to build and project, as an important factor when it comes to achieving job success. In this situation, people become managers of their own brand, entrepreneurs of themselves in the transformed labour market of the 21 st century.

In this way, a new marketing discipline arises, called "personal branding", intended to manage this personal brand. According to theory, the principles of traditional trade can be applied to people, who may be considered as products, but at the same time, as entrepreneurs and directors of their own personal action (subject and object).

This article aims to apply some of the main theories of marketing and branding to the management of the personal brand. It is presented as an exercise to review some of the classic elements and principles of entrepreneurial activity, applicable, today, to the procedures carried out by job seekers. Specific applications of this approach are included for the analysis of the contemporary job market.
\end{abstract}

\section{Keywords:}

Social networks, company self consideration, awareness, brand management, employment, staff selection.

\section{INTRODUCCIÓN: LA IMAGEN PERSONAL Y LA EMPLEABILIDAD EN UN CONTEXTO DE CAMBIO Y COMPETICIÓN.}

Los años transcurridos en España desde el estallido de la crisis de 2008 hasta la actualidad han supuesto un cambio en el paradigma laboral que se venía desarrollando anteriormente (Urraco 2017, caps. 3 y 4). La falta de oportunidades, así como el exceso de demanda de empleo, ha supuesto un cambio de mentalidad para el colectivo de desempleados de nuestro país. En épocas precedentes, el acceso al mercado de trabajo suponía menores esfuerzos para buena parte de la población, dada una mayor oferta de empleo en cualquier sector. Hoy en día, el trabajador ha de buscar su propio autoaseguramiento en un contexto en el que la globalización, la deslocalización, la neorregulación y las nuevas formas de flexibilización del empleo han creado condiciones de mayor incertidumbre y precariedad para los individuos, especialmente para aquellos que más experimentan las demandas de flexibilidad (Cano 2007, Ortiz 2013). 
Por ello, en este contexto, los trabajadores tienden a verse abandonados por un sistema público que se presenta como un mero coach, cuya acción se limita a acciones paliativas puntuales o a operar como garante de las posibilidades de desarrollar las aptitudes personales (Santamaría 2010, 2012). Así, crecientemente, se dibuja un escenario en el que los buscadores de empleo intentan resolver sus necesidades laborales por sí mismos. La creatividad, la imaginación y la innovación constante de estrategias de búsqueda de empleo marca el panorama actual del mercado de trabajo. Resaltar por encima del resto de candidatos se hace ahora más necesario que nunca, por lo que surgen nuevas formas de llamar la atención de las organizaciones privadas, más allá de la utilización de la técnica del currículum en papel. En este sentido, reforzar la imagen del candidato es una de las prioridades que están vigentes actualmente. Con la gran cantidad de currículums que se envían a los centros de trabajo, los selectores de personal no disponen del suficiente tiempo para escoger a qué personas se les realizará finalmente una entrevista. La primera impresión es por tanto fundamental en esta nueva situación, y esta es la filosofía que guía actualmente la búsqueda de trabajo, constituida bajo la égida del paradigma de la activación, que tiene en la noción de "empleabilidad" uno de sus mantras (Santos y Serrano 2006, Alonso 2007, Tovar y Revilla 2012). Y en este paradigma, el trabajador, entendido como un "empresario de sí mismo" (Foucault 2007), ha de gestionar sus capitales, ha de hacerse empleable, aumentando sus posibilidades de participar en el mercado (Gautié 2004; Alonso, Fernández y Nyssen 2009; Muñoz y Santos 2014).

Convertido en comercial de sí mismo (Ehrenreich 2011), objeto y sujeto al mismo tiempo, el individuo tendrá que seguir una lógica de "maximización de sí" (Dubar 2002, p. 148), que tendrá en la gestión de la imagen uno de sus principales elementos de interés. La proyección de una correcta imagen del perfil laboral de los posibles trabajadores se consigue, entre otras cosas, a través de la asociación del nombre con determinados valores juzgados como positivos o deseables. Como si fuesen marcas, los desempleados y, en general, los buscadores de empleo, trabajan constantemente en conseguir que sus datos sean conocidos no sólo con la esperanza de conseguir un puesto de trabajo con unas determinadas condiciones, sino también con la pretensión de que dicho empleo esté bien remunerado y resulte de calidad, según los estándares socialmente establecidos (Cano 2000; Laparra 2006; Prieto, Arnal, Caprile y Potrony 2009). Esta forma de proceder se encuentra cada vez más generalizada entre candidatos y trabajadores y, por ello, la extensión de estas prácticas se está haciendo necesaria en el ámbito laboral de muchos sectores. Para Climent y Navarro (2016), la gestión de marca personal es una tendencia de Recursos Humanos que supone una actualización de la búsqueda de empleo adaptada a la realidad actual, en la que la persona construye su propia identidad con el objeto de ser más atractiva para el mercado laboral. En este proceso se crea un valor adicional que hace al individuo resaltar por encima del resto de candidatos.

Conseguir que el nombre del trabajador se recuerde y se asocie con determinados aspectos puede asemejarse a gestionar una marca propia para diferenciarse del resto de profesionales. Es, por tanto, una tarea muy similar a la que se realiza en las grandes corporaciones a la hora de administrar sus activos intangibles. Esta filosofía ha llegado además a los propios procesos internos de las organizaciones, donde la gestión del renombre personal es una importante tarea para los propios 
trabajadores en la búsqueda de promoción interna. Karaduman (2013) señala que la gestión de la marca personal es relevante también para los ejecutivos de más alto nivel.

Por tanto, podemos hablar de gestión de marca personal, o personal branding, como una disciplina apropiada y necesaria en los actuales procesos de prospección, búsqueda y movilidad laboral, en un contexto sociolaboral caracterizado por la flexibilidad, la incertidumbre y la competitividad, que se aprovecha de toda una serie de desarrollos tecnológicos y de gestión de la mano de obra que actúan como elementos coadyuvantes para la evolución de esta tecnología de manejo de la imagen, del yo laboral de los trabajadores posmodernos.

\section{DESARROLLO TECNOLÓGICO Y PERSONAL BRANDING: LA IMAGEN, LA INFORMACIÓN PERSONAL Y LAS REDES SOCIALES EN PERSPECTIVA HISTÓRICA.}

Toda esta gestión personal e individualizada de la propia imagen/marca del sujeto no podría hacerse sin la existencia de herramientas tecnológicas gratuitas o de bajo coste, que facilitan la realización de estas tareas sin que ello suponga un elevado gasto para la persona. La democratización tecnológica ha brindado la posibilidad de poder desarrollar este tipo de iniciativas y prácticas. Sin un ordenador personal y conexión a Internet es difícil que un candidato pueda redactar un currículum atractivo, una carta de presentación en la que fije sus prioridades y valores, o que pueda enviar un simple correo electrónico a la empresa en la que desea trabajar: la brecha digital se plasma, pues, también en este tipo de cuestiones cotidianas.

Además del acceso a la tecnología, es necesario que los sujetos puedan compartir y comunicar su filosofía de marca personal al resto de candidatos y reclutadores. La creación de Internet supone un impulso para este tipo de procedimientos. Sin embargo, en las primeras etapas del uso de la red no se implementaron herramientas específicas que permitiesen la construcción de la marca personal en el ámbito laboral. Este tipo de herramientas surge con la aparición de la denominada web 2.0, que es la que fomenta la participación del usuario y la creación de contenidos por parte del mismo. Sulé y Prieto (2010) establecen que la web 1.0 es aquella fase de la red que se caracteriza por el uso de páginas estáticas, en las que no participan los usuarios ofreciendo su propio contenido y creaciones, porque solamente acuden a extraer información. En contraposición a este escenario propio de la web 1.0, la web 2.0 se caracteriza por la comunicación en lugar de por basarse únicamente en la información. Así, esta nueva orientación de Internet y sus funcionalidades se basa en que los usuarios puedan aportar la información que deseen a la red, siendo ellos mismos partícipes de la creación de contenidos, en lugar de serlo solamente los desarrolladores habituales y los responsables de las páginas web.

Internet es básicamente información y comunicación. Y es por la existencia de estas dos funciones por lo que, de un modo u otro, surgen las Redes Sociales en la etapa de la web 2.0. Se puede definir la "Red Social en Internet" (a las que a partir de ahora llamaremos simplemente Redes Sociales) como cualquier espacio cibernético de interrelación entre personas. Son páginas, funciones de las mismas o programas donde se puede contactar con otros usuarios (función comunicativa de la red) y obtener e intercambiar documentos, archivos, o simplemente información con esos otros usuarios 
(función informativa de la red). La creación de dichas Redes Sociales marca un nuevo uso de Internet y sirve de base para el desarrollo del personal branding.

En el inicio de la existencia de las Redes Sociales, una gran cantidad de personas que utilizaban la red de redes pasan de utilizar las herramientas que tradicionalmente ofrecían servicios (como pueden ser los portales de Internet, los servicios de descarga de archivos u otros servicios de carácter informativo) a utilizar Internet con sus Redes Sociales como principal herramienta comunicativa. Paralelamente, las grandes empresas dirigen su atención a este ámbito, que investigan para intentar hallar nuevas formas de captar clientes y obtener beneficio.

Con el desarrollo de las Redes Sociales se puede decir que el usuario deja de tomar una actitud pasiva con respecto a las redes para tomar ahora una posición más activa y creadora. Los usuarios ya no son únicamente meros espectadores de la escena, sino que también participan en ella, no sólo creando los contenidos, sino también compartiéndolos con el resto de usuarios. De ahí que la principal funcionalidad que evoluciona en este tipo de nuevo uso sea la comunicativa. Esta comunicación será la que sirva de base para la creación de marcas personales.

Los usuarios se comunican unos con otros, creando y compartiendo información de interés con los demás. De este modo también crean contenidos en la red, a diferencia de la etapa anterior. Además de obtener información también la comparten si creen que es relevante para los demás. Por todo ello, el "intrusismo" del usuario dentro de la propia página es fundamental, ya que es él mismo el que reorganiza y gestiona los contenidos. Estas informaciones manipuladas por los propios usuarios serán el sustento de las campañas de comunicación de marca personal que permitirán a los usuarios de las redes generar un valor diferencial con respecto a otros usuarios. La existencia de estas herramientas gratuitas favorecerá y servirá de sustento a la disciplina del personal branding, ya que, de no existir, los individuos no podrían realizar la comunicación de forma similar a como lo puede realizar cualquier organización con más recursos y capacidades.

Es cierto que Internet ha pasado con el tiempo de ser una mera herramienta informativa a ser una herramienta comunicativa. Pero esta funcionalidad comunicativa no siempre ha estado presente desde la creación de este tipo de herramientas. Así, en las primeras fases de la Red Social, Internet no sólo sirve como herramienta de comunicación, sino como un medio único donde expresar la identidad de cada persona. Si el DNI es el lugar al que nos podemos remitir "oficialmente" para encontrar las características específicas de una persona, como son el sexo, nombre y apellidos, residencia, etc., no resulta un documento en el que pudieran quedar reflejadas la personalidad, el carácter o los gustos y preferencias de dicha persona. Y, sin embargo, este es uno de los principales rasgos o contenidos que poseen las Redes Sociales en su inicio, y el que las hace atractivas para los usuarios. Se puede decir que en este inicio, los usuarios "crean para comunicarse", y la información que importa es la personal de cada usuario, que sirve para dejar constancia de quién se es. Así se entra en contacto con las otras personas que también quedan registradas en las redes y, con ello, los usuarios pueden relacionarse e interactuar.

En estos primeros momentos en el uso de las Redes Sociales, la generación de personas con renombre se hace de forma alejada del ámbito profesional y circunscrita únicamente al ocio individual. En los últimos años es cuando los buscadores de empleo 
han visto una buena oportunidad de adquirir popularidad gracias a la utilización de las Redes Sociales. En este sentido, según Sánchez (2008), podemos preguntarnos cuál es el motivo de que las personas compartan en Internet un contenido elaborado y con calidad, teniendo en cuenta que esto, en apariencia, únicamente redundará en beneficios empresariales para el pequeño grupo empresarial que pueda explotar y utilizar los datos de la Red. La principal ventaja del uso de estas funcionalidades es la utilización de una herramienta de comunicación gratuita que permitirá al usuario crear su propio valor intangible. Por ello, la publicación constante de contenidos relacionados con el perfil laboral del usuario es hoy en día una técnica de gran interés para la disciplina del personal branding.

Estas herramientas permiten al usuario de las tecnologías crear su propio contenido y publicarlo para que pueda ser consultado por cualquier persona interesada. Las Redes Sociales, dado su carácter gratuito, suponen la democratización de las plataformas comunicativas, lo que favorece la creación de su propia marca (Labrecque, Markos y Milne 2011; Xu, Sang y Blasiola 2014, citado en Fernández, Hernández y Sanz-Marcos 2018). Para Karaduman (2013), en ese sentido, las Redes Sociales son el soporte que brinda la oportunidad a los candidatos para comunicarse a sí mismos, aprovechando la gratuidad (o, al menos, la gratuidad inicial) de las herramientas. Del mismo modo, Jiménez (2016) considera que las Redes Sociales son una herramienta de comunicación que ha visto incrementada su importancia a raíz de épocas de crisis pasadas. Para la autora, la marca personal es una disciplina enmarcada en el área de comunicación del marketing, más concretamente considera que es una técnica de Relaciones Públicas.

Es de interés, por tanto, para la investigación de este ámbito, el estudio de las herramientas que permiten a los usuarios realizar una gestión adecuada de la comunicación online de su marca: las Redes Sociales e Internet. Por otro lado, pese a que este enfoque se repite a lo largo de cualquier estrato o grupo social, resultaría especialmente interesante la observación del colectivo de los jóvenes, quizás los participantes del mercado de trabajo más abocados a tener que manejarse en este nuevo escenario de búsqueda de empleo. Anotamos esta cuestión como futura línea de investigación a partir de lo recogido en el presente artículo.

\section{CONCEPTO Y MARCO DEL PERSONAL BRANDING: DELIMITACIÓN DE SUS FUNCIONES EN LAS ORGANIZACIONES Y APLICACIÓN A LA GESTIÓN DE LA MARCA PERSONAL.}

La gestión de las enseñas es fundamentalmente una técnica de comunicación enmarcada dentro del área comercial de las empresas. Si asemejamos la figura del sujeto a un empresario de sí mismo, podremos definir la gestión de marca personal como una herramienta para el desarrollo de actividades de comunicación con sus allegados. Distintos autores definen el personal branding desde este punto de vista con pequeñas matizaciones, como veremos a continuación.

Para Aaker (1991) una marca es un nombre, signo o símbolo que representa bienes, servicios u organizaciones y que se utiliza para distinguirlos del resto de los competidores. Protege a los consumidores a la hora de decidir, ya que diferencia los productos, servicios o empresas de aquellos que pueden parecer o pretenden ser idénticos. A través de la creación de estos signos distintivos las marcas aportan un valor 
intangible a quien las crea y las gestiona. Podemos aplicar esta definición genérica de marca al ámbito del personal branding: la marca personal es un conjunto de signos y símbolos que generan valor para su propietario (Pérez 2012, citado en Climent y Navarro 2016). Si tenemos en cuenta que la disciplina de la gestión de marca es conocida como "branding", podemos decir que el "personal branding" es el proceso de creación y gestión de nombres, signos y símbolos que identifican a una persona en concreto y que generan un valor para esta.

Otros autores, como Karaduman (2013), definen esta disciplina atendiendo únicamente a la utilización de la misma desde el punto de vista de la empleabilidad. De este modo, Karaduman (2013) define el personal branding como un proceso a través del cual los candidatos pueden lanzar sus carreras profesionales, a través de la gestión de la reputación y de la marca personal. Sin embargo, como veremos más adelante, el personal branding no es una técnica que se circunscriba únicamente al ámbito laboral, sino que es una práctica habitual realizada por cualquier individuo, tenga o no carácter profesional.

Como ya hemos mencionado, esta gestión de enseñas es realizada por personas físicas del mismo modo que lo hacen las entidades, imitando actitudes, valores, objetivos y comportamientos de las grandes organizaciones. Para Montoya y Vandeheley (2002), la marca personal va más allá de la simple imagen personal, ya que es un ámbito de carácter estratégico, en el que los individuos toman decisiones a largo plazo con respecto a su público objetivo, segmentación y acciones concretas que tienen una meta dirigida (Montoy y Vandehey 2002, citado en Jiménez 2016).

Por tanto, es necesario enmarcar la gestión de las marcas dentro de las organizaciones, para comprender cuál es la función de la misma en el ámbito de la gestión personal. Realizaremos, por tanto, a continuación, una comparativa entre la labor de las organizaciones y la de cualquier persona que adopte las premisas del personal branding.

Para diversos autores, la estructura de las empresas se puede dividir en distintas áreas o subsistemas de acuerdo con la actividad que realicen. De este modo, delimitamos canónicamente cuatro subsistemas que representan las principales tareas agrupadas de las entidades privadas: el subsistema de marketing/comercial, el subsistema financiero, el subsistema de operaciones/fabricación y el subsistema de administración (Barroso 2014, Gutiérrez 2016). Cada una de estas divisiones tendrá un cometido diferenciado. El área administrativa es la encargada de la gestión de los recursos, de la documentación y de los trabajadores de la empresa. Algunos autores, como Gutiérrez (2016), separan el área administrativa de la de los Recursos Humanos, que se encargará, de forma autónoma, del reclutamiento, selección, motivación, formación y capacitación del personal de las organizaciones. Precisamente, el demandante de empleo deberá dirigirse a esta sección empresarial para realizar sus acciones de comunicación.

El área financiera será la encargada de la búsqueda de recursos económicos destinados a la inversión y al gasto. El área de operaciones, como su propio nombre indica, estará dirigida a la realización y organización de sus actividades rutinarias relacionadas con la fabricación o venta de los productos. Por último, el subsistema comercial y de marketing será el que establezca las estrategias de comercialización y 
venta, así como las que tienen que ver con las relaciones de las organizaciones con sus grupos de interés.

Del mismo modo que podemos definir las áreas funcionales de las organizaciones, podemos aplicarlas a la actividad diaria de un demandante de empleo que pretenda hacer de sus tareas un trabajo en sí mismo.

El sujeto deberá gestionar su propia documentación, llevar un registro y clasificación adecuados para que su trabajo sea fructífero, de la misma manera que se practica en el área de administración de una entidad. Por otro lado, también deberá destinar recursos para la consecución de las tareas que le llevarán a encontrar un empleo, trabajo que se asemeja al que se realiza en el área financiera de una empresa. Como señala Karaduman (2013), es necesaria una adecuada estructura financiera para poder hacer crecer la notoriedad o la lealtad de marca. Las marcas son activos intangibles que tienen un valor económico empresarial. Este valor no es fruto de la casualidad, sino del esfuerzo financiero que realizan las grandes organizaciones para hacer crecer este bien. Los recursos utilizados pueden ser de cualquier índole: tiempo, bienes o incluso recursos económicos. El individuo que realice este tipo de tareas debe decidir invertir sus ahorros o más o menos escasos ingresos no sólo en imprimir currículums, sino que deberá disponer de la tecnología adecuada (móvil, ordenador, o incluso otros dispositivos, como tablets). Además, en algunos casos (y como veremos en los siguientes apartados), los sujetos deben decidir invertir recursos económicos en una mayor publicidad y presencia de sí mismos en la red. El buscador de empleo puede, pues, realizar las actividades propias del área de operaciones de una organización, si bien en este caso se trata de un servicio que no está dirigido a ningún otro cliente, sino a sí mismo.

Como venimos comentando, las tareas más importantes de las realizadas por el buscador de empleo son las que relativas a comercialización y marketing. El individuo es administrador de su propia labor comercial. Dentro de este área podemos identificar algunas funciones que realizan las personas, y que se asemejan cada vez más a las llevadas a cabo en las organizaciones. Tomaremos como referencia la visión del marketing que proponen Kotler y Amstrong (2012):

Dirección estratégica de marketing. Como cualquier organización en la realización de su trabajo, los individuos deben fijar previamente unos objetivos estratégicos (generalistas y a corto plazo) que marquen la meta principal de su actividad. Además, el individuo deberá tener clara cuál es su misión (su razón de ser como profesional), su visión (cómo quiere verse como profesional en el futuro) y los valores que le llevarán hasta ese punto. Por último, deberá elaborar un plan temporal de acción, en el que se especifique de manera concreta qué tácticas (acciones concretas, a corto plazo) va a realizar para alcanzar las metas marcadas.

Función de investigación del mercado. El mercado, o los posibles "clientes" que contratarán los servicios de los demandantes de empleo, serán las empresas interesadas o las que hayan publicado con anterioridad una oferta de empleo. Se convertirán en clientes en el momento en el que decidieran incorporar al trabajador en su actividad habitual. El sujeto será el responsable de realizar prospecciones en la búsqueda de clientes potenciales, así como de facilitar una comunicación con los mismos. Por otro lado, dentro del estudio de su posible mercado, determinará quiénes pueden ser sus principales competidores, para poder hacer un estudio comparativo de su 
perfil con respecto al de estos. Con toda esta información podrá tomar las decisiones más adecuadas para alcanzar los objetivos marcados previamente.

- $\quad$ Definición de las tácticas de marketing. Para Luca, Ioan y Sasu (2015), cualquier profesional, independientemente de su perfil profesional o especialización, debe realizar campañas de marketing que le permitan poder ser elegido por consumidores $\mathrm{u}$ otros profesionales. Tomando como referencia la estructura utilizada en el ámbito de la comercialización, los sujetos pueden realizar prácticas similares a la hora de definir su perfil profesional. Esta estructura es conocida como "las $4 p$ del marketing" o "el mix de marketing", y divide las decisiones en cuatro ámbitos: el del producto (product), el del precio (price), el de la distribución (placement) y el de la comunicación (promotion). Veremos qué implicaciones directas tiene este enfoque en cada una de estas áreas:

- Área de producto. El individuo se convierte en un bien de intercambio, que debe ser vendido a los empleadores y que puede ser utilizado en un proceso productivo o comercial para obtener una utilidad o un rendimiento. Podemos estar hablando, por tanto, de un producto con carácter industrial, ya que su uso o consumo es de utilidad en la creación de otros bienes o servicios. El empleador será "comprador" o, visto de otro modo, será arrendatario de los servicios que preste este producto. En cualquier caso, el sujeto se cosifica, se convierte en un objeto de mercancía con un precio.

Dentro de las decisiones que se pueden tomar con respecto al producto podemos nombrar aquellas que tienen que ver con su diseño, características, funcionalidades, embalaje, etiquetado, o con la marca. Como productos y, a su vez, como responsables de sí mismos en cuanto a la estrategia de comercialización, los trabajadores y desempleados deberán decidir cuáles serán sus características diferenciadoras con respecto al resto de candidatos. Deben decidir qué ofrecerán que les caracterice, en cuanto a su funcionalidad (por ejemplo, las tareas que pueden desempeñar), características (por ejemplo, su formación y capacitación) o incluso en cuanto a su marca. Enmarcaremos, por tanto, el ámbito del personal branding dentro del área de decisiones en cuanto al individuo como producto.

- $\quad$ Área de precio. El demandante de empleo, en este caso, en la mayoría de las ocasiones decidirá cuál es la remuneración o rendimientos que deberá obtener por el alquiler de sus servicios como producto. Será el empleador el que, observando sus características o el valor que le reportará, asigne finalmente una cantidad, que el demandante aceptará o rechazará. Esta es una peculiaridad del mercado de trabajo con respecto a un mercado de bienes o consumo, ya que el comprador es el que fija el precio de la transacción y no la entidad fabricante o comercializadora. Esto se produce por el elevado número de oferentes (de desempleados) que se encuentran en búsqueda activa de empleo. En situaciones en las que al empleador (el cliente, en este caso) le es dificultoso encontrar un candidato que se ajuste a sus requerimientos, el oferente tendrá un mayor poder en la toma de decisiones sobre la cantidad (salario) a asignar a su trabajo (el servicio).

- Área de distribución. En este punto debemos hablar acerca de decisiones sobre el canal de distribución, la participación de intermediarios, almacenamiento y logística de mercancías. Al tratarse de personas, y no de objetos que pueden ser distribuidos y almacenados, los demandantes de empleo no deberán tener en cuenta 
estas variables de cara a su estrategia de marketing. Solamente deberán tener en cuenta cuál será su área geográfica de interés a la hora de desarrollar, llegado el caso, su actividad laboral.

- Área de comunicación. En este punto, el sujeto deberá determinar cómo contactar a su público de interés, qué técnicas utilizará, los canales y soportes, así como también el mensaje que desea transmitir. Para la generación de una correcta campaña comunicativa los individuos deben tener en cuenta lo definido en la dirección estratégica de marketing, así como el contenido de la investigación de mercado previa, ya que de ello dependerá la coherencia del conjunto. Sobre todo, será fundamental la revisión de las decisiones tomadas en cuanto a marca (el objetivo primordial del presente artículo) para generar una correcta campaña comunicativa dentro de las decisiones de planificación de marketing. Tomaremos como referencia Internet como el principal canal de comunicación en la generación del valor de marca, y las Redes Sociales como el soporte más importante.

Una vez delimitado el ámbito en el que el personal branding se enmarca, podemos profundizar en la generación del valor de marca personal para los trabajadores y demandantes de empleo. Cuestión que será tratada de un modo relativamente exhaustivo en el siguiente apartado.

\section{GENERACIÓN DE VALOR DE MARCA PERSONAL: ACTUACIONES A TRAVÉS DE SUS COMPONENTES.}

Los productos, además del valor que les corresponde por sus características físicas, presentan, en ocasiones, un valor adicional asociado a atributos intangibles de los mismos, tal y como puede ser la marca. Este valor generado puede ser valorado según distintos puntos de vista. Una de las aportaciones más aceptadas en el ámbito del marketing es la de Keller (1993), que propone que el valor de marca sea definido a partir de las reacciones que el consumidor experimente hacia la misma. A este tipo de valoración Keller (1993) la define como "valor capital de marca basado en el cliente" (customer-based Brand equity). Para este autor, el valor de marca en sí mismo es el conjunto de reacciones que la marca provoca en los consumidores cuando son expuestos a las acciones de marketing (López 2017a, 2017b). De este modo, con la visión que propone el autor, analizamos la marca siempre desde el punto de vista del cliente. Esta opción de valoración de enseñas es muy apropiada para la disciplina del personal branding, utilizada por el público interesado en el producto (trabajador), en este caso los empleadores, reclutadores o responsables de RR.HH. de las organizaciones. Sus opiniones y valoraciones sobre el candidato serán las que formen el valor de marca personal de los sujetos en el ámbito laboral.

Por otro lado, en lugar de valorar la marca personal desde una perspectiva que sólo se basara en las opiniones de los interesados en las mismas, se puede tener en cuenta lo que aporta la marca, por sí misma, al trabajador. Esta visión de valor es la que propone Aaker (1991) cuando define la marca como un conjunto de elementos que suman o restan valor, en última instancia, al producto. De este modo, podemos decir que, hoy en día, una mala gestión del personal branding puede suponer la disminución del valor último del candidato en una primera revisión rápida por parte de los reclutadores. De igual modo, una correcta gestión de las premisas del personal branding asegura un incremento del valor primario del candidato, por lo que las posibilidades de éxito en un proceso selectivo se verían aumentadas. Las premisas del personal branding 
obligan, por tanto, a que los individuos creen un sistema comunicativo de marca a nivel profesional, ya que la no existencia de una estrategia en este sentido puede suponer que su valor de mercado como producto se vea mermado, y, por tanto, que no tengan posibilidades de éxito en sus pretensiones.

Aunando estas definiciones, podemos decir que el valor de la marca personal será, en suma, el conjunto de reacciones positivas y negativas que el nombre de una persona, entendido como marca, provoca en el público interesado en la misma. Las personas, por ello, si quieren conseguir reacciones que sean de su interés, deberán trabajar en la construcción de este valor. Para realizar una correcta administración de marca por parte de los sujetos es necesario tener en cuenta que el valor de marca se forma a partir de componentes que, sumados, son los que articulan la importancia de la enseña personal.

Como se explica en trabajos previos (López 2017b), existen distintas formas de estructurar el valor de marca, como la que proponen Dyson, Farr, y Hollis (1996). Sin embargo, la mayor parte de las investigaciones del ámbito del marketing se basan en las ya mencionadas propuestas de Keller y de Aaker, que serán las que utilicemos a continuación.

Cada uno de los autores indicados define distintos componentes del valor que podemos revisar para aplicarlos al ámbito de la creación de valor de marca personal. Una vez hecho este análisis podremos reunir ambas teorías y definir unos componentes de valor de marca unificados para la gestión del personal branding.

Aaker (1991) propone cuatro factores que integran el valor de marca. Estas cinco dimensiones se subdividen en diez medidas distintas que pueden ser utilizadas por el interesado para hacer una valoración de su capital de marca (López 2017a, 2017b). Estos componentes, aplicados al ámbito del personal branding, son:

- $\quad$ Lealtad de marca. Contar con gran cantidad de clientes fieles incrementa el valor de las enseñas, por lo que será un importante objetivo para el personal branding el de asegurar una masa crítica de interesados en la persona que exponga su nombre como marca. Una de las técnicas utilizadas por los individuos con marcas personales renombradas consiste en ofrecer contenidos constantes y de interés a través de las Redes Sociales. Con esta estrategia, los responsables de la marca personal consiguen gran cantidad de seguidores interesados en la temática de los contenidos compartidos, que asocian con determinados valores de la marca personal. Este público constante, que está al tanto de las novedades expuestas, suele estar compuesto por los responsables de Recursos Humanos de las distintas organizaciones. Es importante, por tanto, que el público objetivo de la estrategia de marca (reclutadores, selectores de personal) sea fiel y leal a estos contenidos, ya que la comunicación constante hacia los mismos en modo publicitario asegura que recuerden la marca personal y puedan contar con el individuo en los diferentes procesos selectivos o cuando exista una necesidad concreta.

En este sentido, el personal branding alcanza su máximo exponente en la figura de los llamados "líderes de opinión" o "influencers" que han conseguido formar una base de seguidores amplia a lo largo del tiempo y que tienen asegurado el éxito comunicativo en las redes. Según Fernández et al. (2018), los líderes de opinión han sido individuos con mejor y mayor acceso a las plataformas de comunicación, situación que últimamente ha ido cambiando de forma sustancial. Estos gestores de marca personal son, por tanto, la figura a la que quiere llegar cualquier persona que esté interesada en que su marca personal alcance el éxito en el mundo profesional. 
Como conclusión, podemos decir que más que conseguir una clientela fiel en términos de compra del producto, lo que pretende la estrategia de lealtad de marca en personal branding es conseguir un conjunto amplio de individuos atentos a las novedades comunicativas. Si el sujeto es finalmente contratado o ascendido por parte de la organización la estrategia de lealtad dejaría de tener sentido si fijamos la atención solamente en la adquisición (incorporación o promoción laboral). La gestión de marca personal profesional debería ser continuada a lo largo del tiempo, por lo que el incremento del valor a través de la lealtad se adquirirá, sobre todo, a través de un correcto marketing de contenidos en Redes Sociales.

Las medidas que propone Aaker (1991) para alcanzar la lealtad son la satisfacción del cliente y el precio superior que este está dispuesto a pagar por la marca (López 2017b). En el ámbito del personal branding, al considerar clientela a la audiencia de este marketing de contenidos, el gestor de marca personal deberá intentar que el conjunto de receptores de información esté satisfecho con los contenidos ofrecidos. Si existe un deseo de contratación, también existirá el de pagar un precio superior (salario) por los servicios del trabajador. A mayor satisfacción de la audiencia, y mayor deseo de pagar por sus servicios, se producirá un incremento de la lealtad a la marca y, por ende, un incremento del valor de la marca personal.

Notoriedad de marca. Para Aaker, la notoriedad de marca es la importancia que tiene la misma en la mente del consumidor. Esta se mide a través del reconocimiento, conocimiento y el recuerdo que tiene el consumidor de la misma, así como a través de otros factores, como si la marca es la primera que se puede recordar cuando le preguntan por marcas del sector y si es la única marca que puede recordar o la recuerda junto con otras, o la opinión que tiene de la misma (López 2017b).

Así, observamos que el recuerdo y el conocimiento que tengan los consumidores de las marcas es un elemento fundamental en la construcción de la notoriedad, por lo que las Redes Sociales, en este caso, son una herramienta que puede favorecer la creación de notoriedad en el caso del personal branding. El marketing de contenidos, además de asegurar un conjunto de interesados en las comunicaciones de los trabajadores o desempleados, servirá de herramienta que fomente la repetición del nombre de las personas. El objetivo de realizar publicaciones periódicas y constantes por parte del individuo es el de aparejar su nombre al ámbito tratado en dichas publicaciones. La simple repetición de noticias, artículos o informaciones de interés hará que el nombre de un candidato aparezca de forma constante e ininterrumpida, y además contextualizada, en su sector laboral. Esto incrementará el recuerdo y el conocimiento del candidato por parte de los seleccionadores de las organizaciones, suponiendo una ventaja competitiva para el candidato con respecto al resto de competidores.

En un mundo cada vez más globalizado e interconectado, no es de extrañar que los buscadores de empleo se afanen en conseguir contactos no sólo de reclutadores de empresa, sino también de profesionales de su mismo sector que puedan abrirles puertas en caso de existir una oportunidad.

- Asociaciones de marca. Son los valores que los consumidores e interesados asignan a las enseñas y que representan su esencia final. Estas asociaciones se encuentran ligadas a los dos valores que hemos definido anteriormente (lealtad y 
notoriedad), ya que, en el caso del personal branding, podemos conseguir asociar atributos a través del marketing de contenidos.

Esta técnica, además de conseguir un mayor recuerdo y conocimiento del candidato, así como de favorecer la existencia de un grupo de interesados leales, producirá por parte del que recibe la información relaciones inconscientes con aspectos profesionales del candidato. Por ello, cuidar la naturaleza y orientación de las publicaciones, artículos, informaciones, noticias o contenido multimedia es relevante para el gestor de su propia marca. Desviar la atención del público a temas que no interesen o no encajen con su perfil laboral producirá confusión en los receptores de la información. El mensaje lanzado por el candidato debe ser claro, uniforme y coherente para que el recuerdo por repetición sea factible y la asociación con los valores deseados se fije finalmente en la mente del que reciba la comunicación.

No es de extrañar que el personal branding deba incidir en la idea de que la marca corresponde a un individuo, antes que confundir al interesado haciéndole creer que se trata de una empresa o de un producto de consumo. Dicho de otro modo, el nombre de la marca personal no debe ser otro que el nombre de la propia persona. Además, en lugar de utilizar un logotipo, sería recomendable utilizar siempre una fotografía del candidato o trabajador. Con estas pequeñas tácticas aseguramos que la audiencia reconozca la marca como perteneciente a una persona, y que la asociación marca-persona se produzca de una manera satisfactoria. Utilizar otra denominación o un logotipo esconde a la persona que se encuentra detrás de la misma, y no consigue llevar a cabo estrategias de fidelización, recuerdo y asociaciones que sean satisfactorias.

- $\quad$ Calidad percibida. Se trata del último de los componentes que propone Aaker, y responde a la calidad percibida y liderazgo de la marca. Forma, junto con la lealtad, un bloque de gran importancia en la generación de valor. Al igual que ocurre con la lealtad, marca el límite en cuanto al precio que el reclutador estaría dispuesto a pagar por la incorporación del individuo en la plantilla de la empresa (López 2017b).

Una manera adecuada de generar mayor calidad percibida será la actualización constante de los contenidos que ofrece el sujeto, así como también de su perfil laboral de cara a su audiencia. Un adecuado perfil expuesto en Redes Sociales profesionales supondrá un incremento del valor de marca personal. Este currículum online, a raíz de la publicación de contenidos por parte del usuario, será visitado de forma frecuente por parte de los empleadores, que serán los que acaben fijando el valor final del candidato. Podemos decir que, generalmente, asociar el contenido del currículum online con empresas u organizaciones de reconocido prestigio contribuirá a mejorar la calidad percibida y las asociaciones.

Otras cuestiones, como la coherencia de la carrera profesional, el detalle en las explicaciones sobre los trabajos anuales (sin que existan períodos de vacío sin explicación aparente), el detalle en la relación de las tareas llevadas a cabo en los puestos de trabajo, una descripción adecuada o la inclusión de informaciones referentes a las actividades de voluntariado (si así se considerara), pueden ser aspectos generales que contribuyan a una mejora en la percepción de la calidad del perfil y, por tanto, del candidato.

Por su parte, Keller (2008), dentro de su visión del valor capital de marca como técnica de valoración de enseñas, también divide este valor en varios componentes y medidas fundamentales (López 2017b). En este caso, el autor diferencia seis piezas 
fundamentales, que organiza en una pirámide de construcción de valor de marca. Estas, a su vez, se organizan en distintos escalones dentro de la propia pirámide, llegando a diferenciarse cuatro pasos o fases en la construcción del valor de marca a partir del consumidor, como las que especificaremos a continuación:

- $\quad$ Construcción de la identidad de marca y de la prominencia de la misma. En este caso, la marca debe percibirse por parte del consumidor como incluida y apropiada para una categoría de producto. Para ello es necesario que el que recibe las informaciones asocie la marca con determinados valores. La prominencia de marca es un componente que puede asemejarse al de notoriedad expuesto por Aaker. Como Keller incluye las asociaciones en este concepto, podemos decir que asociaciones y notoriedad son conceptos muy similares. Como ya hemos explicado, es necesario que los trabajadores y candidatos trabajen para que sus campañas comunicativas estén orientadas en un único sentido, a fin de que su audiencia encasille su perfil rápidamente en un sector concreto.

- $\quad$ El siguiente paso en el proceso de construcción del valor es aquel en el que el consumidor dota de significado a la propia marca. Puede corresponderse con las opiniones que se generan en la audiencia antes de probar el propio producto en cuestión. Para ello, el consumidor del producto valorará, a simple vista, si el producto es adecuado para satisfacer una necesidad concreta. Keller diferencia entre las valoraciones que el consumidor hace desde un punto de vista objetivo y subjetivo. Las valoraciones objetivas las hará a través del desempeño de marca o, lo que es lo mismo, a través del análisis de las características reales del producto, como su diseño, precio, eficiencia... Las valoraciones subjetivas las hará en base a sus propias características personales.

Para el gestor de su marca personal es importante tener bien definido su perfil laboral en las Redes Sociales. Esta es la principal herramienta para mostrar sus características como producto, el documento que muestra todas sus posibilidades y elementos que pueden ser utilizados por las organizaciones. Como hemos mencionado anteriormente, el perfil que el candidato mantiene en las Redes Sociales se convierte en su DNI laboral, en la hoja de características en la que los responsables de selección de las entidades pueden encontrar las especificaciones del producto. Es por tanto necesario mantener un currículum digital actualizado que acompañe a las publicaciones periódicas y relacionadas con su perfil. El reclutador será el que haga las valoraciones en base a este perfil online. A partir de este juicio se generará un incremento o una disminución del valor de marca, y en ello influirá de manera definitiva el mantenimiento del currículum digital. Esta técnica será la que maneje las valoraciones subjetivas de la audiencia sobre la marca personal del candidato. Para manejar las valoraciones subjetivas de su público, el interesado deberá conectar con la personalidad o experiencias pasadas de los seleccionadores, utilizando una vez más las publicaciones que se elaboran con el marketing de contenidos. Por ello, además de intentar conseguir asociaciones de marca, prominencia/notoriedad y calidad percibida, el marketing de contenidos servirá de ayuda para manejar las valoraciones subjetivas que dotarán de significado a la marca personal.

- Una vez que el consumidor identifica la identidad de la marca y la dota de un significado, en el siguiente nivel se produce la respuesta del consumidor hacia la marca. En este momento se producen los juicios del consumidor con respecto a la 
enseña (respuesta objetiva), así como los sentimientos hacia la marca (respuesta subjetiva) (López 2017b). Según Aaker (2008), la valoración de la calidad es una respuesta objetiva a la identidad y el significado de la marca, por lo que podríamos asociar este componente al de calidad percibida que define el propio Aaker (1991).

El sentido de las publicaciones, así como el tono que el candidato muestre en su perfil profesional, puede producir juicios y sentimientos positivos o negativos hacia la marca personal por parte de su audiencia. Es necesario, por ello y una vez más, que el responsable de su marca cuide al máximo sus contenidos y que estos sean coherentes, para favorecer asociaciones oportunas que fijen juicios y sentimientos positivos en la mente de su público objetivo.

- $\quad$ Es en el último paso en el que se fija la relación final del consumidor con respecto a la marca. En este punto es cuando se determinará la implicación de la audiencia con respecto al candidato y trabajador y donde se comprobará si es fiel, o no, a la marca personal y los contenidos del individuo. Es, por tanto, una fase semejante al componente de lealtad a la marca que define Aaker (1991). En este punto es donde el candidato tendrá referencia sobre el éxito de su trabajo. Valorará si consigue finalmente gran cantidad de seguidores de sus contenidos y si llega a obtener un importante número de contactos y personas interesadas en su perfil. Esta puede ser la medida final de obtención de valor de marca personal, ya que el objetivo último de esta técnica es conseguir contactos que abran nuevos y prometedores caminos profesionales al que la utiliza.

Expuestas las dos principales teorías sobre valor de marca, trataremos de aunar ambas para explicar el proceso de creación de valor de marca personal en el ámbito profesional.

\section{PROCESO DE CREACIÓN DEL VALOR DE MARCA PERSONAL Y PROFESIONAL: UNA APROXIMACIÓN A LAS BUENAS PRÁCTICAS EN EL PERSONAL BRANDING.}

Como hemos visto anteriormente, la creación de una marca personal con valor suficiente para que este sea el adecuado en el mercado de trabajo no es una cuestión menor ni sencilla. El responsable de la marca que le identifica debe dedicar tiempo y recursos que favorecerán el éxito de la estrategia. Por norma general, el comportamiento de los individuos se asemejará al de una organización con plena capacidad comercial, por lo que sus actuaciones no sólo se limitarán a publicitarse, sino que deberán estar atentos al resto de actuaciones de sus competidores y deberán, también, plantear estrategias. Después de esto podrá tomar decisiones concretas en cuanto a su propia marca.

Teniendo en cuenta todo lo expuesto, podemos delimitar las siguientes fases de creación de valor de marca personal y profesional, pensando qué esfuerzos debe realizar el candidato en cada una de las etapas para conseguir alcanzar sus objetivos.

1. Investigación del mercado laboral y delimitación de las líneas estratégicas. Lo primero que debe hacer el candidato es concretar su sector de actuación. Cuanto más concreto sea, más efectiva será su campaña comunicativa, siempre teniendo en cuenta que tendrá el inconveniente de tener un perfil poco flexible y adaptable. Ser 
demasiado rígidos definiendo puestos ideales puede cerrar puertas a otras posibles oportunidades.

Una vez definido el sector estratégico al que vamos a dirigir los esfuerzos será necesario que el candidato busque qué perfiles, actuaciones y posicionamiento aplican el resto de competidores (el resto de los candidatos). Normalmente, Internet suele ser el recurso de referencia para la búsqueda de otros candidatos, ya que la red será la principal herramienta utilizada por todos ellos. En concreto, el desarrollo de las Redes Sociales profesionales no solamente facilita la labor de búsqueda e investigación, sino que también ofrece un espacio virtual específico para mantener ordenado y visible el currículum de los trabajadores. Estos portales, gratuitos y de fácil registro y uso, son los que han ampliado las posibilidades de generación de valor para los sujetos particulares. Además, las Redes Sociales generalistas, no centradas en el ámbito profesional, también complementan el contenido de estas últimas. Se convierten, por tanto, en un espacio informal en el que se pueden volcar informaciones relacionadas con la profesión o sector de los candidatos.

Con la investigación del mercado de su sector laboral, los individuos pueden buscar sugerencias, ideas o actuaciones creativas del resto de competidores, ideas que pueden adaptar a sus propias estrategias. Todo ello contribuirá a la creación de un conocimiento general del funcionamiento del mercado de trabajo en su sector que marcará sus actuaciones posteriores en la generación de valor.

También en esta fase se establecerán las principales líneas estratégicas, como si el buscador de empleo fuese una organización en sí mismo, definiendo la misión, visión y los valores con los que operará en su campaña. Asimismo, marcará los objetivos que quiere conseguir: un empleo, un ascenso, conseguir más contactos o simplemente incrementar su popularidad como profesional del sector.

2. Determinación de las tácticas de marketing. En este caso, el candidato deberá seguir las premisas del marketing mix (las "4 p") para esclarecer las líneas generales de creación de valor. Será necesario que el candidato pueda definirse como producto, determinando cuáles serán sus características diferenciales con respecto a los competidores: capacidades, habilidades, conocimientos, formación, experiencia, etc. También deberá delimitar su precio, entendiendo como tal la banda salarial en la que el candidato se encuentra actualmente, o en la que pretende encontrarse con posterioridad. Por último, dentro de la estrategia de comunicación, tendrá que determinar, antes de comenzar con su estrategia de marcas, el medio o los medios de comunicación que le servirán de soporte en sus actuaciones. Ya hemos expuesto que Internet y las Redes Sociales, a raíz de la aparición de la web 2.0, son las herramientas más elegidas para construir la marca personal.

3. Posteriormente, el trabajador o candidato deberá comenzar a crear la identidad de marca, el cuerpo central de la misma. Lo hará, en un primer momento, con la creación de su perfil profesional en Redes Sociales, y la inclusión en las mismas de un eslogan efectivo elaborado con las palabras clave de su sector. Con ello, el candidato busca adquirir prominencia y notoriedad inicial de su nombre en su categoría profesional. Un perfil laboral en redes que esté completo en cuanto a cantidad y exhaustividad de información favorece que los individuos sean encontrados por los reclutadores o selectores de personal, y que sean identificados como referentes dentro 
de su área. Como complemento, las publicaciones de contenidos relacionados favorecerán el conocimiento y notoriedad de la marca.

4. El público objetivo de la campaña, a partir del contenido que ofrezca el sujeto y de las asociaciones que comiencen a crear en sus mentes, llevará a dotar de significado a la marca. Las valoraciones objetivas y subjetivas realizadas por la audiencia harán que se produzca finalmente un posicionamiento en sus mentes y es donde podremos observar si la fijación de la identidad de marca se ha hecho de forma efectiva o no. Si los interesados en el candidato entienden el significado final de la identidad que se pretendía transmitir, la marca personal verá incrementada su valoración.

5. A partir de los juicios y sentimientos que la audiencia haga del significado de la marca personal se determinará, de forma inconsciente, qué relación final va a mantener la audiencia con esa persona, es decir, con un sujeto entendido como un producto con marca. En este punto es donde el candidato podrá evaluar el desempeño de su campaña de comunicación con respecto a los objetivos marcados. Si al cabo de un determinado período temporal (fijado previamente en las primeras fases de la generación del valor de marca) el candidato consigue un empleo, debe seguir manteniendo los contactos o el reconocimiento fijados como metas al inicio de la campaña, para seguir manteniendo la marca a lo largo del tiempo. Recordemos que según Aaker (1991) las actuaciones con respecto al valor de marca pueden resultar un conjunto de elementos que sumen o resten ese valor. La inacción o la no publicación de contenidos en las redes restarán valor de marca personal al individuo una vez conseguidos los objetivos. Por otro lado, si el candidato observa que las metas no han sido conseguidas, deberá reiniciar la estrategia desde el primer paso.

\section{CONCLUSIONES: LA CRECIENTE IMPORTANCIA DE LA GESTIÓN DE LA MARCA PERSONAL EN EL INCIERTO (Y ACELERADO) MERCADO DE TRABAJO DEL SIGLO XXI.}

Podemos afirmar que el personal branding, o la gestión de la marca personal, es una disciplina de nueva creación que se ha implantado en el mercado laboral, convirtiendo a los empleados y trabajadores en productos con una marca que coincide con su propia identidad. Dicho de otro modo, el nombre propio de los sujetos se convierte en un bien intangible con un valor construido a partir de una estrategia profesional de gestión de marcas.

La aparición de este nuevo enfoque se produce por varios motivos. Las escasas oportunidades laborales del contexto español poscrisis, marcado por el exceso de oferta y la escasez de demanda de mano de obra, obligan a los desempleados y trabajadores a buscar nuevas fórmulas para intentar destacar por encima de otros profesionales de su sector. Además, el desarrollo de la tecnología y, específicamente, de Internet, permite un mayor y mejor contacto entre empresas y trabajadores. La evolución en el uso de la red por parte de los usuarios ha provocado la aparición de la web 2.0, que es un enfoque novedoso en la utilización de la misma. En este nuevo sistema, los usuarios pasan de ser meros consultores de información a ser sujetos generadores de los contenidos. Su necesidad de participación activa en Internet ha provocado que las páginas web dejen de ser estáticas y de estar directamente controladas por las empresas para pasar a ser un 
elemento dinámico en el que los propios usuarios marcan el ritmo y contenido de sus publicaciones.

Con estas premisas, la disciplina de la marca personal se ha implantado de manera clara en el conjunto de participantes del mercado laboral. Tanto oferentes como demandantes de empleo consideran de obligado cumplimiento sus postulados, ya sea de manera consciente o inconsciente. Aunque el usuario no sepa de la profesionalización de sus actividades, en la práctica está realizando una actividad muy similar a la que realizan los trabajadores del área de comunicación de cualquier organización lucrativa.

Esta filosofía de mercantilización del individuo es la que sustenta el personal branding. Para construir una marca personal con valor, el sujeto deberá poner en práctica las técnicas de creación de un currículum online (o perfil profesional virtual) y las de publicación constante de contenidos en las Redes Sociales profesionales. Deberá realizar ambas técnicas con un enfoque de coherencia, en el que busque que cualquier actuación realizada se relacione con el ámbito o perfil profesional al que desea pertenecer. La audiencia de este tipo de comunicaciones (reclutadores, selectores de personal o compañeros de profesión) es la que sumará o restará valor a la marca personal del individuo, a través de las asociaciones (más o menos acertadas) que los miembros de dicha audiencia hagan en sus mentes. Por ello, podemos afirmar que el valor de marca personal en el ámbito profesional será un valor basado siempre en el cliente, en línea con los postulados del valor de marca que define Keller (2008).

El proceso de creación de este valor pasa por varias fases. Después de definir sus metas como profesional, en un primer momento el candidato realizará un estudio de la situación del mercado de trabajo en el sector al que quiere pertenecer. Tras estructurar un breve plan de marketing, donde definirá sus características diferenciales como producto, elaborará un currículum virtual ligado a los contenidos temáticos que publique, intentando con ello crear una identidad concreta con notoriedad, posicionamiento y prominencia en las mentes de los interesados en su perfil. Este público acabará finalmente por dotar de significado a la marca de manera inconsciente.

Si el responsable de la marca consigue que su nombre se asocie con su sector de referencia, y que dicho nombre tenga el significado para el público que inicialmente se deseaba, habrá conseguido una estrategia de personal branding satisfactoria. Para llegar a esto, el candidato deberá haber dedicado el esfuerzo y los recursos necesarios propios de una actividad similar realizada en cualquier empresa a la hora de conseguir una clientela fiel.

\section{REFERENCIAS BIBLIOGRÁFICAS}

AAKER, D. Managing brand equity. Nueva York: Simon and Schuster, 1991. ISBN 0-02-900101-3

ALONSO, L.E. La crisis de la ciudadanía laboral. Barcelona: Anthropos, 2007. ISBN 978-84-7658-817-8

ALONSO, L.E., FERNÁNDEZ, C.J. y NYSSEN, J.M. El debate sobre las competencias: una investigación cualitativa en torno a la educación superior y el mercado de trabajo en España. Madrid: Agencia Nacional de Evaluación de la Calidad y Acreditación, 2009. ISBN 978-84-691-7394-7 
BARROSO, C. Economía de la empresa. Madrid: Pirámide, 2014. ISBN 97884-368-2740-8

CANO, E. Análisis de los procesos socioeconómicos de precarización laboral. En Cano, E., Bilbao, A. y Standing, G., Precariedad laboral, flexibilidad y desregulación. Alzira: Germania, 2000, pp. 25-68. ISBN 978-84-89847-25-5

CANO, E. La extensión de la precariedad laboral como norma social. En: Sociedad y Utopía: Revista de Ciencias Sociales, 2007, nº 29, pp. 117-137. ISSN 1133-6706

CLIMENT J.A. y NAVARRO, Y. Nuevos retos en orientación laboral: de itinerarios personales de inserción a la construcción de marcas profesionales. En: Revista Española de Orientación y Psicopedagogía, 2016, v. 27, $\mathrm{n}^{\mathrm{o}}$ 2, $2^{\circ}$ Cuatrimestre, pp. 126-133. ISSN 1989-7448

DUBAR, C. La crisis de las identidades: la interpretación de una mutación. Barcelona: Bellaterra, 2002. ISBN 978-84-7290-185-8

DYSON, P., FARR, A. y HOLLIS, N. S. Understanding, measuring, and using brand equity. En: Journal of Advertising Research, 1996, vol. 36, $n^{\circ}$ 6, pp. 9-22. ISSN 0021-8499

EHRENREICH, B. Sonríe o muere: la trampa del pensamiento positivo. Madrid: Turner, 2011. ISBN 978-84-7506-938-8

FERNÁNDEZ, J. D., HERNÁNDEZ-SANTAOLALLA, V. y SANZ-MARCOS, P. Influencers, marca personal e ideología política en Twitter. En: Cuadernos.info, 2018, no 42, pp. 19-37. ISSN 0719-3661

FOUCAULT, M. Nacimiento de la biopolítica: curso en el Collège de France (1978-1979). Buenos Aires: Fondo de Cultura Económica, 2007. ISBN 978-950-557715-6

GAUTIÉ, J. Repensar la articulación entre mercado del trabajo y la protección social en el postfordismo. En: Cuadernos de Relaciones Laborales, 2004, v. 22, $\mathrm{n}^{\circ} 1$, pp. 147-184. ISSN 1131-8635

GUTIÉRREZ, O. Fundamentos de la dirección de empresas. Madrid: Pirámide, 2016. ISBN 978-84-368-36523

KARADUMAN, I. The effect of social media on personal branding efforts of top level executives. En: Procedia-social and behavioral sciences, 2013, v. 99, pp. 465473. ISSN 1877-0428

KELLER, K.L. Administración Estratégica de Marca. Branding. México: Pearson Educación, 2008. ISBN 978-970-26-1284-1

KELLER, K.L. Conceptualizing, measuring, and managing customer-based brand equity. En: Journal of Marketing, 1993, v. 57, nº 1, pp. 1-22. ISSN 0022-2429

KOTLER, P. y AMSTRONG, G. Fundamentos de marketing. México: Pearson Educación, 2012. ISBN 978-607-32-1420-9

LABRECQUE, L.I., MARKOS, E. y MILNE, G.R. Online personal branding: Processes, challenges, and implications. En: Journal of interactive marketing, 2011, vol. 25, no 1, pp. 37-50. ISSN 1094-9968

LAPARRA, M. La construcción del empleo precario: dimensiones, causas y tendencias de la precariedad laboral. Madrid: Cáritas / Fundación FOESSA, 2006. ISBN 978-84-8440-367-8

LÓPEZ, S. La notoriedad y calidad percibida de la marca del distribuidor como fuentes del valor de marca para el establecimiento. Badajoz: Universidad de Extremadura (Tesis doctoral inédita), 2017b. 
LÓPEZ, S. Relaciones entre componentes del valor del distribuidor y sus marcas. En: URRACO, M., GALLARDO, D. y LÓPEZ, S. (eds.), Catálogo de investigación joven en Extremadura, v. 1, Cáceres: Servicio de Publicaciones de la Universidad de Extremadura, 2017a, pp. 193-196. ISBN 978-84-608-9677-7.

LUCA, F.A., IOAN, C.A., y SASU, C. The Importance of the Professional Personal Brand. The Doctors' Personal Brand. En: Procedia Economics and Finance, 2015, v. 20, pp. 350-357. ISSN 2212-5671

MONTOYA, P. y VANDEHELEY, T. The brand called you: Make your business stand out in a crowded marketplace. New York: McGraw-Hill, 2009. ISBN 978-007-15-9750-0

MORALES, M.J. Relaciones públicas y redes sociales: creación e implementación de marcas personales. En: Opción: Revista de Ciencias Humanas y Sociales, 2016, $\mathrm{n}^{\circ}$ 9, pp. 945-960. ISSN 1012-1587

MUÑOZ, D. y SANTOS, A. Hoy es el futuro. De la activación universitaria a las respuestas colectivas frente a la precariedad juvenil. En: Revista de la Asociación de Sociología de la Educación, 2014, v. 7, no 3, pp. 658-673. ISSN 1988-7302

ORTIZ, P. Flexibilidad laboral en el mercado de trabajo español. En: Áreas: Revista Internacional de Ciencias Sociales, 2013, n 32, pp. 93-102. ISSN 0211-6707

PÉREZ, A. Marca personal. Como convertirse en la opción preferente. Madrid: ESIC, 2012. ISBN 978-847-35-6557-8

PRIETO, C. (Coord.), ARNAL, M., CAPRILE, M. y POTRONY, J. La calidad del empleo en España: una aproximación teórica y empírica. Madrid: Ministerio de Trabajo e Inmigración, 2009. ISBN 978-84-8417-348-9

SÁNCHEZ, J.R. Perspectivas de la información en Internet: ciberdemocracia, redes sociales y web semántica. En: Zer-Revista de Estudios de Comunicación, 2008, v. 13, no 25, pp. 61-81. ISSN 1137-1102

SANTAMARÍA, E. "Buscarse la vida": trayectorias y experiencias de precariedad en el acceso al empleo de las personas jóvenes. En: Revista de Estudios de Juventud, 2010, no 89, pp. 101-123. ISSN 0211-4364

SANTAMARÍA, E. Jóvenes y precariedad laboral: trayectorias laborales por los márgenes del empleo. En: Zerbitzuan, 2012, nº 52, pp. 129-139. ISSN 1134-7147

SANTOS, A. y SERRANO, A. Presentación. El giro copernicano del desempleo actual. En: Cuadernos de Relaciones Laborales, 2006, v. 24, $\mathrm{n}^{\circ}$ 2, pp. 1-19. ISSN 11318635

SULÉ, M.A. y PRIETO, J. MK-20 Secretos a voces del social media. En: Pecvnia, Monográfico Studia Mercatoria Legionensia. Miscelánea de Marketing (Monográfico), 2010, pp. 191-214. ISSN 2340-4272

TOVAR, F.J. y REVILLA, J.C. La institucionalización de la individualización del trabajo: el concepto de flexiguridad. En: Cuadernos de Relaciones Laborales, 2012, v. 30, $\mathrm{n}^{\mathrm{o}} 1$, pp. 235-258. ISSN 1131-8635

URRACO, M. "Un saco de niños zaleados": precariedad laboral y precariedad vital de la "generación de la crisis" en Extremadura. Madrid: Universidad Complutense de Madrid (Tesis doctoral inédita), 2017.

XU, W.W., SANG, Y., BLASIOLA, S., y PARK, H.W. Predicting opinion leaders in Twitter activism networks: The case of the Wisconsin recall election. En: American Behavioral Scientist, 2014, v. 58, nº 10, pp. 1278-1293. ISSN 1552-3381 